\title{
Image-Guided Radiotherapy Dose Escalation in Intermediate and High Risk Cancer Prostate Patients and Its Effect on Treatment Toxicity
}

\author{
Mohsen S. Barsoum1, Azza Mohamed Nasr'1, Ikram Hamed Mahmoud², Salem E. Salem³, \\ Rasha A. Elawady ${ }^{1}$, Shaimaa Abdelallem4, Ahmed Awad ${ }^{1 *}$ \\ ${ }^{1}$ Department of Radiation Oncology, NCI-Cairo University, Cairo, Egypt \\ ${ }^{2}$ Department of Radio Diagnosis, NCI-Cairo University, Cairo, Egypt \\ ${ }^{3}$ Department of Medical Oncology, NCI-Cairo University, Cairo, Egypt \\ ${ }^{4}$ Department of Medical Statistics, NCI-Cairo University, Cairo, Egypt \\ Email: *ahmed.abdallah@nci.cu.edu.eg
}

How to cite this paper: Barsoum, M.S., Nasr, A.M., Hamed, I.M., Salem, S.E., Elawady, R.A., Abdelallem, S. and Awad, A. (2017) Image-Guided Radiotherapy Dose Escalation in Intermediate and High Risk Cancer Prostate Patients and Its Effect on Treatment Toxicity. Journal of Cancer Therapy, 8, 591-602.

https://doi.org/10.4236/jct.2017.86050

Received: May 13, 2017

Accepted: June 24, 2017

Published: June 27, 2017

Copyright $\odot 2017$ by authors and Scientific Research Publishing Inc. This work is licensed under the Creative Commons Attribution International License (CC BY 4.0).

http://creativecommons.org/licenses/by/4.0/

\begin{abstract}
Purpose: To study the effect of escalating radiation dose; in intermediate and high risk prostate cancer patients; via online image-guidance on acute toxicities. Patients and Methods: thirty-eight prostate cancer patients were treated by using simultaneous integrated boost-intensity modulated radiation therapy (SIB-IMRT) with online image guided correction via kilo voltage cone beam computed tomography (KV-CBCT)/electronic portal imaging device (EPID) of trans-rectal ultrasound (TRUS)-inserted intraprostatic gold fiduciary markers. High-risk patients received a median dose of 80.5 Gy to prostate and 56 Gy to pelvic nodes in 35 fractions over 7 weeks. Intermediate-risk patients received a similar prostate dose over the same overall treatment time. Acute toxicity (bladder, rectal and bowel symptoms) was reported once weekly during the radiation course and up to 3 months from the end of the radiation course. Results: The image guided (IG)-IMRT allows escalating the radiation dose delivered to the prostate through minimizing the margin of setup error to less than $0.5 \mathrm{~cm}$ with subsequent sparing of nearby organs at risk. Out of thirty-eight patients, no patient developed > grade 1 acute rectal toxicity, $7.9 \%$ of patients experienced grade 3 urinary toxicity and there was no reported small intestinal toxicity. Conclusion: Escalating the radiation dose more than $80 \mathrm{~Gy}$ in intermediate and high risk prostate cancer patients was safe and not associated with grade 3 - 4 RTOG toxicity when guided by online verification of intra-prostatic fiducial markers.
\end{abstract}

\section{Keywords}

Prostate Cancer, High-Risk, Intermediate-Risk, Fiducial Markers, 
Image Guided Radiotherapy, Dose Escalation, Acute Toxicity, SIB-IMRT

\section{Introduction}

Prostate cancer radiotherapy is a constantly evolving field. The large number of sensitive adjacent structures (rectum, bladder, penile bulb, and femoral heads), the mobility and distension of the rectum and the gradual filling of the bladder make the conformal treatment of the prostate gland quite a challenging process. The geometric uncertainties are made complex by the fact that the nearby critical structures can move and change shape both relative to bony anatomy and to each other [1] [2]. Imaging methods such as portal imaging or radiographic imaging allow localization of bony anatomy but do not permit characterization of the changes in shape and relative position of the gland and surrounding normal tissues. The use of markers inserted in the gland provides a significant improvement in gland-targeting accuracy and precision over bony anatomy thus allowing minimizing the margin employed in the planning target volume (PTV) [3]-[9]. Technical advances such as dose-escalated radiotherapy (RT) and intensity modulated radiotherapy (IMRT) have allowed more conformal and efficient treatment of tumour with low risk of morbidity. Image-guided radiotherapy (IGRT) with daily imaging and repositioning can increase the efficacy and safety of therapy by maximizing the accuracy and precision of therapy and minimizing the risk of treatment-related toxicity [6].

\section{Patients and Methods}

This is a prospective phase II study including intermediate and high risk prostate cancer patients who were presented to the National Cancer Institute (NCI), Cairo University, between June 2015 and December 2016. All patients had adenocarcinoma of the prostate diagnosed by trans-rectal ultrasound-guided (TRUS) core needle biopsy and categorized as having an intermediate or high risk disease according to risk grouping system proposed by D'Amico et al. (1998) and fulfilling the following inclusion criteria:

1) ECOG Performance Scale (0 - 2).

2) Adequate liver functions.

3) Adequate renal function (serum creatinine $\leq 1.5 \mathrm{mg}$ ).

4) No evidence of distant metastasis or other malignancy.

5) Informed consent.

6) Patient must be accessible for treatment and follow up.

7) No previous history of pelvic radiation.

All eligible patients were subjected to complete history and physical examination, laboratory investigations including total prostatic specific antigen (PSA), complete blood count, blood urea nitrogen and serum creatinine, radiological investigations including chest X-ray, magnetic resonance imaging (MRI) pelvis, Computed tomography (CT) abdomen and bone scan. 
All patients received 2 months of neoadjuvant hormonal treatment with total androgen blockade either by subcapsular orchiectomy or luteinizing hormone releasing hormone (LHRH) agonist combined with antiandrogens. The patient then underwent TRUS and 3 fiducial markers (gold seeds) are inserted into the prostate ( 2 in the base of the gland; one on either side in the peripheral zone; and one in the apex). One week later (waiting for any possible inflammation to resolve), CT planning and MRI pelvis (T2 study for fusion) are performed. Patients were simulated while lying supine. Planning CT is performed with comfortably full bladder (by voiding then drinking $600 \mathrm{cc}$ of water half an hour before simulation) and empty rectum (by rectal enema done the day before simulation; at night). The patients are instructed to reproduce the simulation position during each fraction (by drinking the same amount of water before each fraction and eating high fibre diet together with laxatives to ensure adequate rectal emptying).

The treatment volume varies according to the risk group. In intermediate risk patients, the proximal one $\mathrm{cm}$ of the seminal vesicles is treated to a dose of $56 \mathrm{~Gy}$ with the entire prostate receiving the $80.5 \mathrm{~Gy}$. In high risk patients, the entire prostate received the full dose with the pelvic nodes included in the volume receiving 56 Gy. The whole length of the seminal vesicles is included in the PTV 56 unless grossly infiltrated where it is included in the PTV 80.5. The entire prostate is delineated as the clinical target volume (CTV) with the aid of the MRI (T2 weighted images) with special attention to the prostate apex (the transition between the prostate apex and genito-urinary diaphragm [GUD] is accurately demarcated on MRI). An expansion of 0.7 centimetre $(\mathrm{cm})$ in all directions except posteriorly where it is $0.5 \mathrm{~cm}$ is put around the CTV prostate to generate the PTV 80.5. Pelvic lymph nodes (including the distal common iliac, internal iliac, external iliac, obturator and presacral lymph nodes[LN]) are delineated ;guided by the corresponding vessels with $0.7 \mathrm{~cm}$ margin around the vessel excluding the surrounding muscle, bone and bowel with presacral lymph nodes extending from S1-S3; to create the CTV LN. An expansion of $0.5 \mathrm{~cm}$ around the entire seminal vesicles and around the CTV LN is put to generate the PTV 56 excluding the adjacent areas of PTV 80.5. Organs at risk are delineated including the bladder, rectum, penile bulb, head of femurs and bowel bag.

Radiation is delivered over 35 fractions to two different volumes using SIB technique with 2.3 Gy per fraction to the PTV 80.5 and 1.6 Gy per fraction to the PTV56. All patients were treated by IMRT using inverse treatment planning system (TPS; Monaco, Elekta). A homogenous distribution to the treatment volume with maximum deviation of $+7 \%$ and $-5 \%$ is ensured. The plan is evaluated by two-step process; by evaluating the isodose lines covering the target cut by cut then evaluating the dose volume histogram (DVH) for both the target and nearby normal tissues. The patient received 35 fractions; 5 fractions per week with at least once weekly online verification of the fiducial marker position using CBCT or EPID. After finishing the radiation course, high risk patients should continue hormonal treatment for a total period of 2 years whereas the interme- 
diate risk patients continue for 2 more months.

All patients were seen once weekly during the radiation course then monthly with reporting up on the acute toxicity (bladder, rectal and small bowel toxicity). The radiation therapy oncology group (RTOG) acute Radiation Morbidity Schema was used to score radiation toxicities appearing during and up to 90 days from the end of radiation course.

\section{Results}

Forty-three patients fulfilled the inclusion criteria. Five patients were excluded from the study due to different reasons. Two patients achieved mild response to neoadjuvant hormonal treatment (initially presented with locally advanced T4 disease) with subsequent inevitable unacceptable high bladder dose so they were excluded from the dose escalation protocol and received the conventional dose. Three patients lost follow up for reasons not related to the disease. One patient developed fracture of the left femur following fiducial marker insertion and was not able to present to NCI for further management. One patient received 6 fractions then developed cerebrovascular stroke and have a gap of one and half month then presented to us for further management. All investigations were repeated (PSA, MRI pelvis and bone scan) and revealed localized disease. The patient continued radiation treatment but he was excluded from the study. One patient received 21 fractions then died after car accident. So we analyzed 38 patients out of the 43 accrued patients.

Their ages ranged between 56 and 84 years. The mean age for the entire group was $68.1 \pm 7.2$ years with a median age of 66 years. PSA was measured initially at diagnosis for each patient with its value $\leq 20 \mathrm{ng} / \mathrm{ml}$ in 7 patients (18.4\%) and $>20$ $\mathrm{ng} / \mathrm{ml}$ in 31 patients ( $81.6 \%$ of the entire cohort). For the entire cohort, Gleason score ranged between 4 and 10 with a mean of $6.95 \pm 1.2$ and median of 7. Patients classified as having high risk disease were 33 patients representing $86.8 \%$ while those classified as having intermediate risk disease were only 5 patients representing 13.2.\% of the entire cohort as per D'Amico grouping system [10] (Table 1).

It is worth mentioning that the overall treatment time ranged from 45 to 98 days with a mean of $60.4 \pm 12.3$ and a median of 57 days (this was largely attributed to the machine breaks). Out of the thirty-eight evaluable patients, twenty-five patients (65\% of the entire cohort) had at least once weekly online imaging with six patients (out of these twenty-five) had twice weekly imaging. The remaining thirteen patients had between 3 - 6 times online verification during the entire course. EPID was used only when the CBCT was out of function. This online imaging was used to accurately verify the position of the three implanted intraprostatic fiducial markers. Matching is done between the online image and the planning CT image in three directions; vertical, horizontal and longitudinal. The resulting errors are corrected and the patient is treated. The mean of the detected error was $0.36 \mathrm{~cm}$ in the vertical direction, $0.36 \mathrm{~cm}$ in the horizontal direction and $0.48 \mathrm{~cm}$ in the longitudinal direction (Figure 1). 
Table 1. Distribution of patients according to different sociodemographic and clinical characteristics.

\begin{tabular}{ccc}
\hline Patient characteristic & Sub-category & Number of patients \\
\hline Age group in years & $51-60$ & 9 \\
& $61-70$ & 14 \\
& $71-80$ & 14 \\
PSA & $>80$ & 1 \\
& $\leq 20 \mathrm{ng} / \mathrm{ml}$ & 7 \\
& $>20 \mathrm{ng} / \mathrm{ml}$ & 31 \\
& 4 & 2 \\
Gleason score & 5 & 2 \\
& 6 & 6 \\
& 7 & 20 \\
Risk group & 8 & 3 \\
& 9 & 4 \\
& 10 & 3 \\
\hline
\end{tabular}

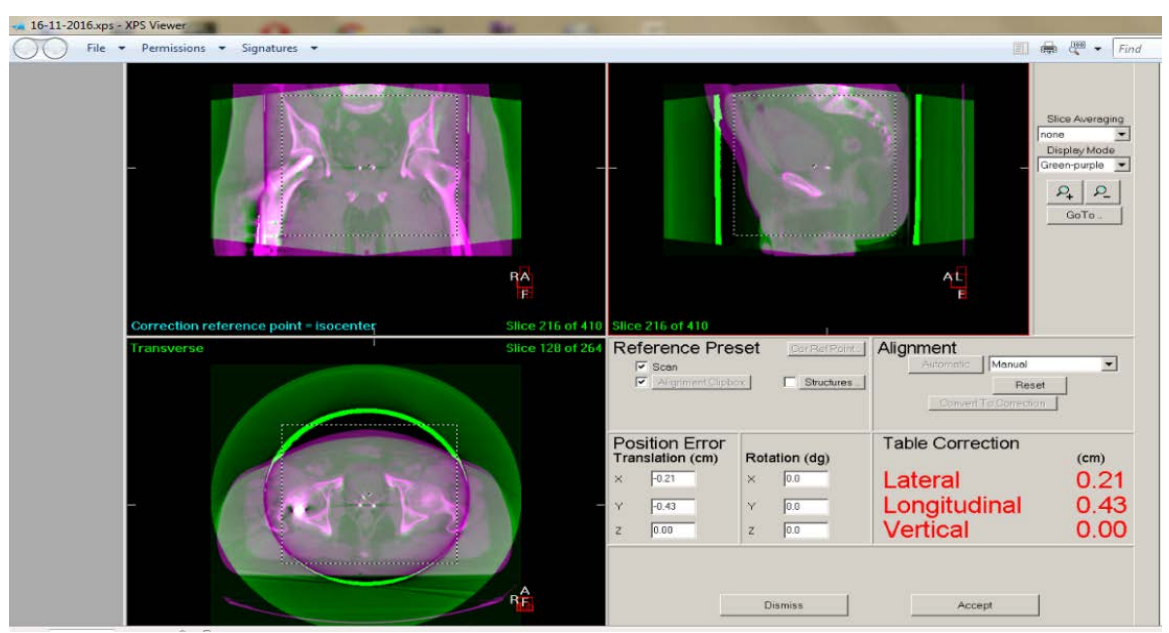

Figure 1. Online verification of 3 intraprostatic fiducial markers by using KV-CBCT showing the resulting error.

The patients who developed $\leq$ grade 2 RTOG urinary toxicity were 34 out of the 38 evaluated patients representing $89.4 \%$ of the entire cohort while those who developed >grade 2 toxicity were 3 patients representing $7.9 \%$. One patient has inserted urinary catheter throughout the radiation course and was not candidate for the evaluation criteria of the RTOG toxicity. Patients were prescribed supportive treatment including pain killers and urinary antiseptics.

Thirty-two patients; out of the 38 evaluated patients; developed grade 0 RTOG rectal toxicity (84.2\%) while those who developed grade 1 toxicity were 6 patients (15.8\%). One patient; out of these six patients; developed inflamed exter- 
nal piles with severe tenesmus persisting for 3 months after the end of radiation course with no response to the usual medications and improved only upon nerve block. Four patients received supportive treatment and improved during the radiation course while the remaining one improved 2 months after the end of the radiation treatment. There was no reported RTOG rectal toxicity greater than grade I within the entire cohort.

Doses delivered to the bowel bag, femoral heads and penile bulb are far below the tolerance doses and there were no symptoms suggestive of treatment related toxicities affecting these organs.

\section{Discussion}

Radiation therapy (RT) is widely used in the treatment of prostate cancer [11] [12]. Dose escalation has been adopted in radical radiotherapy of prostate cancer to make full use of its advantage of improved tumour control outcomes [13]-[20]. The current "standard" dose of primary RT for prostate cancer is now between 75 and $80 \mathrm{~Gy}$, which is $10 \%$ to $20 \%$ higher than what can be safely delivered with conventional RT techniques [14]. Since many patients diagnosed with non-metastatic prostate cancer have life expectancy longer than 10 years, the choice of RT techniques with minimized radiotherapy-related toxicity is crucial for improving quality of life [15] [21]. However, higher doses have raised the concern of increased normal tissue toxicity, such as gastrointestinal (GI) toxicity and genitourinary (GU) toxicity [13] [22].

In the past two decades several technological innovations have advanced the planning and delivery of RT, thereby allowing for dose escalation to a prostate target while sparing the nearby organs at risk with subsequent reduction in radiation induced toxicity. Insertion of fiducial markers within the prostate; which can be tracked via online imaging (By CBCT or EPID); has made it easy to accurately target and deliver the radiation dose to the prostate by minimizing the margin of setup error. These benefits have now been demonstrated in several prospective randomized trials [16] [20] [22] [23]. IMRT is increasingly becoming the most used radiation technique for prostate cancer, providing higher doses to both the prostate and the pelvic LN with optimized dose distributions and with decreased volumes of nearby critical organs receiving high doses. Moreover, IMRT allows the safe delivery of simultaneous integrated boost (SIB) to any microscopically or macroscopically involved area [24].

Saracino et al. reported the clinical results in 110 patients with high-risk prostate cancer. Thirty seven patients presented with localized prostate cancer and radiological evidence of node metastases or $\geq 15 \%$ estimated risk of lymph node (LN) involvement, while 73 patients underwent postoperative adjuvant or salvage irradiation for biochemical or residual/recurrent disease, LN metastases, or high risk of harbouring nodal metastases. Treatment is delivered using pelvic IMRT and SIB to the prostate area. The treatment volume included the pelvic lymph nodes (common, internal, external iliac and presacral LNs) together with prostate and seminal vesicles. The reproducibility of the treatment was daily ve- 
rified by using orthogonal portal images [24].

Similarly in the present study, we used pelvic IMRT with SIB to the prostate area and our treatment volume included the pelvic lymph nodes, prostate and seminal vesicles (in high risk patients). The reproducibility of treatment was done by online verification of intraprostatic fiducial markers using at least once weekly cone beam CT (CBCT).

The rate of patients free from G2 acute urinary, rectal and intestinal toxicities in Saracino et al. was $61 \%, 60 \%$, and $77 \%$ of patients respectively in comparison to $84.2 \%, 100 \%$ and $100 \%$ in the present study. None experienced G3 toxicities in Saracino et al. On the contrary, 3 patients in the present study; representing $7.9 \%$ of the entire cohort; developed grade 3 or more urinary toxicity.

The RTOG 0126 prostate cancer trial; which compared conventional dose (70.2 Gy) radiation therapy to dose escalated (79.2 Gy) conformal radiation therapy in the management of intermediate risk prostate cancer; has reported on the acute toxicity. This trial was initiated with 3DCRT and amended after 1 year to allow IMRT. IMRT patients were treated to $79.2 \mathrm{~Gy}$. The treatment volume included the prostate and proximal $1 \mathrm{~cm}$ of seminal vesicles in a single phase treatment [25]. In the present study, the intermediate risk patients represented only $13 \%$ of the entire cohort with the remaining $87 \%$ being high risk. While the treatment in the RTOG study was a single phase with one volume, we employed 2 volumes treated simultaneously using SIB technique. The $1^{\text {st }}$ volume included the prostate and proximal $1 \mathrm{~cm}$ of seminal vesicles and was treated to $56 \mathrm{~Gy}$. The $2^{\text {nd }}$ volume included the prostate only and received the full dose of $80.5 \mathrm{~Gy}$.

When analyzing the acute toxicity, the IMRT-treated patients in the RTOG study experienced a $9.7 \%$ rate of $\geq$ grade 2 acute GI and/or GU toxicity in comparison to $13 \%$ rate of acute GU toxicity with no acute grade 2 or more GI toxicity in the present study. This may be attributed to a larger treatment volume in the present study (by including the pelvic nodes in high risk cases) with subsequent higher bladder dose. Also, the use of fiducial markers in our study allows for a tighter posterior margin with subsequent sparing of the rectum [25].

Arcangeli studied the treatment-related morbidity in 55 patients with prostate cancer who were treated with an optimized pelvic IMRT and SIB to prostate or prostate bed. 24 patients received a radical radiation therapy and the remaining 31 patients received a postoperative irradiation as adjuvant treatment or after biochemical or macroscopic local or regional relapse. The treatment volume included the prostate, seminal vesicles and pelvic lymph nodes. In the radical irradiation group, the prescription dose to prostate and seminal vesicles was progressively escalated from $74-76 \mathrm{~Gy}$ (7 pts), to 76 - $78 \mathrm{~Gy}$ (6 pts), and to $79-80$ Gy (11 pts) in 2 Gy fractions. Pelvic lymph nodes were treated in the same number of fractions to a total dose ranging from 51 to 59 Gy (equivalent to 45 50 Gy given in 2 Gy fractions)The accuracy of set-up was daily verified by orthogonal portal images. Similarly in the present study, the treatment volume included the prostate, seminal vesicles and pelvic lymph nodes (pelvic lymph nodes were not treated in intermediate risk patients). The prescription dose to 
prostate was 80.5 Gy over 35 fractions in 2.3 Gy fractions (Equivalent to 86.5 in 2 Gy fractions by using a/ß ratio of 2 ). Pelvic lymph nodes and seminal vesicles; unless grossly infiltrated; were treated in the same number of fractions to a total dose ranging from 56 Gy (equivalent to 50 Gy given in 2 Gy fractions). Treatment setup verification was done guided by the implanted fiducial markers using at least once weekly CBCT.

In Arcangeli study, Patients without acute grade 2 intestinal, rectal, and GU toxicity were $91 \%, 71 \%$, and $63 \%$ respectively in comparison to $100 \%, 100 \%$ and $89.4 \%$ respectively in the present study [26]. This may be attributed to a better online verification method (CBCT in the present study as compared to orthogonal portal imaging in Arcangeli study).

Spratt conducted a study comparing tumour control and toxicity outcomes with the use of high-dose IMRT alone or brachytherapy combined with IMRT (combo-RT) for patients with intermediate-risk prostate cancer. The patients treated with IMRT alone (470 patients during the period from 1997-2010) received a dose of 86.4 Gy in 48 fractions of $1.8 \mathrm{~Gy}$. The PTV consisted of the prostate, seminal vesicles (pelvic lymph nodes were not included) plus $1-\mathrm{cm}$ margin in all directions except posteriorly, where the margin was $0.6 \mathrm{~cm}$. Patient position was verified with weekly port films. Less than $5 \%$ of patients had fiducial marker insertion for image-guided radiotherapy in this cohort [27]. In the present study, The PTV consisted of the prostate, seminal vesicles (pelvic lymph nodes were included in high risk disease) plus $0.7 \mathrm{~cm}$ margin in all directions except posteriorly, where the margin was $0.5 \mathrm{~cm}$. Patient position was verified with at least weekly CBCT/EPID. All patients had 3 implanted intraprostatic fiducial markers.

The rectal toxicity reported by Spratt was similar to that observed in the present study while the urinary toxicity was slightly better as $8 \%$ of patients in the present study developed grade 3 toxicity (compared to $0.4 \%$ in Spratt et al.). This may be explained by larger treatment volume used in the present study; in order to treat the pelvic lymph nodes; as compared to a more localised volume in Spratt study.

Wortel et al. selected prostate cancer patients treated with 3D-CRT $(n=215)$ and IG-IMRT $(\mathrm{n}=260)$ receiving $78 \mathrm{~Gy}$ in 39 fractions within 2 randomized trials in Erasmus Medical Center Cancer Institute, Rotterdam and Netherlands Cancer Institute for toxicity reporting. Within IG-IMRT group, all patients were prescribed 78 Gy to the prostate with image-guided daily on-line set-up verification and correction with implanted fiducial markers and CBCT were in $96 \%$ of patients. For the remaining 4\%, an off-line image-guided protocol using kilovoltage CBCT imaging was used for prostate matching. Off-line bony anatomy matching with electronic portal imaging was used for 3D-CRT [28].

Acute GI toxicity $\geq$ RTOG grade 2 was reported in $29 \%$ of patients within the IG-IMRT cohort in Wortel et al. as compared to $16 \%$ with $\geq$ RTOG grade 1 toxicity (no reported grade 2 toxicity) in the present study whereas acute GU toxicity $\geq$ RTOG grade 2 was experienced by $38 \%$ of patients as compared to $13 \%$ in the present study. 
Rudat et al. has reported on the interfractional change of prostate position and on the acute toxicity using IG-IMRT in localised prostate cancer with a median total dose was 79.2 Gy (range 77.4 - 81.0 Gy). Similar to the present study, 3 fiducial gold markers were inserted into the prostate guided by TRUS. They inserted the seed in the prostate base, mid gland and the apex while we put 2 seeds in the base and 1 in the apex. Online verification was performed using EPID while we used KV-CBCT and EPID (when CBCT was out of function). The treatment volumes were similar including the prostate and seminal vesicles for intermediate risk patients and adding pelvic lymph nodes for high risk patients [29]. The combined error was more or less the same ranging from $4-5 \mathrm{~mm}$ in Rudat et al. as compared to $3.5-4.5 \mathrm{~mm}$ in the present study. The frequency of online verification was better in Rudat et al. with EPID done on daily basis as compared to once or twice weekly KV-CBCT in the present cohort.

The urinary toxicity in Rudat et al. was analyzed using the common terminology criteria for adverse events (CTCAE) v.4.03 and revealed no grade 3 or 4 acute reactions. This is slightly better than the present study as $8 \%$ of patients developed grade 3 urinary toxicity. This may be explained by the more frequent use of online verification in Rudat et al. allowing for tighter margin with subsequent reduction in doses delivered to nearby critical structures.

The inter-fraction change of prostate position is a major concern in treatment of prostate cancer patients. Skarsgard et al. has measured the required CTV-PTV margin a counting for this change in order to reduce the risk of geographic miss. They reported that without image guidance, margins of $0.57 \mathrm{~cm}, 0.79 \mathrm{~cm}$ and $0.77 \mathrm{~cm}$, along the left-right, superior-inferior and anterior-posterior axes respectively, are required to give $95 \%$ probability of complete CTV coverage each day. With image guidance (daily EPID), these margins were reduced to $0.36 \mathrm{~cm}$, $0.37 \mathrm{~cm}$ and $0.37 \mathrm{~cm}$ respectively. These margins are comparable to those observed in the present study which was detected by at least once weekly CBCT. The margins detected in the present study were $0.36 \mathrm{~cm}, 0.48 \mathrm{~cm}$ and $0.36 \mathrm{~cm}$ along the left-right, superior-inferior and anterior-posterior axes respectively [30].

\section{Conclusion}

Using dose escalation more than $80 \mathrm{~Gy}$ for prostate cancer treatment to get the reported clinical benefit can be implemented safely. When delivered by SIBIMRT and using image guidance strategy guided by online verification of intra-prostatic fiducial markers with at least once weekly CBCT. This permits the use of narrower CTV to PTV margins, and smaller PTV volume without compromising the coverage of the target with maximum sparing of critical organs. This will lower the incidence of the acute GI or GU toxicity with the use of IG-IMRT.

\section{Recommendation}

1) Extending the follow up for this cohort of patients up to 3 years is required to report the late toxicity and to prove the clinical benefit of dose escalation. 
2) Once weekly online verification using KV-CBCT/EPID does not compromise the precision of radiation delivery as the reported mean of inter-fraction error; less than $0.5 \mathrm{~cm}$; was comparable to more frequent online verification.

\section{References}

[1] Crook, J.M., Raymond, Y., Salhani, D., et al. (1995) Prostate Motion during Standard Radiotherapy as Assessed by Fiducial Markers. Radiotherapy and Oncology, 37, 35-42. https://doi.org/10.1016/0167-8140(95)01613-L

[2] Ghilezan, M.J., Jaffray, D.A., Siewerdsen, J.H., et al. (2005) Prostate Gland Motion Assessed with Cine-Magnetic Resonance Imaging (Cine-MRI). International Journal of Radiation Oncology, Biology, Physics, 62, 406-417.

https://doi.org/10.1016/j.ijrobp.2003.10.017

[3] Balter, J.M., Kwok, L.L., Sandler, H.M., et al. (1995) Automated Localization of the Prostate at the Time of Treatment Using Implanted Radiopaque Markers: Technical Feasibility. International Journal of Radiation Oncology, Biology, Physics, 33, 1281. https://doi.org/10.1016/0360-3016(95)02083-7

[4] Balter, J.M., Lam, K.L., Sandler, H.M., et al. (1994) Automated Tracking of Prostate Position Using On-Line Portal Imaging. Medical Physics, 21, 951.

[5] Balter, J.M., Sandler, H.M., Lam, K., et al. (1995) Measurement of Prostate Movement over the Course of Routine Radiotherapy Using Implanted Markers. International Journal of Radiation Oncology, Biology, Physics, 31, 113-118. https://doi.org/10.1016/0360-3016(94)00382-U

[6] Chung, P.W., Haycocks, T., Brown, T., et al. (2004) On-Line Portal Imaging of Implanted Fiducial Markers for the Reduction of Interfraction Error during Conformal Radiotherapy of Prostate Carcinoma. International Journal of Radiation Oncology, Biology, Physics, 60, 329-334. https://doi.org/10.1016/j.ijrobp.2004.03.038

[7] Nederveen, A., Lagendijk, J., Hofman, P., et al. (2000) Detection of Fiducial Gold Markers for Automatic On-Line Megavoltage Position Verification Using a Marker Extraction Kernel (MEK). International Journal of Radiation Oncology, Biology, Physics, 47, 1435-1442. https://doi.org/10.1016/S0360-3016(00)00523-X

[8] Vigneault, E., Pouliot, J., Laverdiere, J., et al. (1997) Electronic Portal Imaging Device Detection of Radioopaque Markers for the Evaluation of Prostate Position during Megavoltage Irradiation: A Clinical Study. International Journal of Radiation Oncology, Biology, Physics, 37, 205-212. https://doi.org/10.1016/S0360-3016(96)00341-0

[9] Nichol, A.M., Brock, K.K., Lockwood, G.A., et al. (2007) A Magnetic Esonance Imaging Study of Prostate Deformation Relative to Implanted Gold Fiducial Markers. International Journal of Radiation Oncology, Biology, Physics, 67, 48-239. https://doi.org/10.1016/j.ijrobp.2006.08.021

[10] D’Amico, A.V., Whittington, R., Malkowicz, S.B., et al. (1999) Pretreatment Nomogram for Prostate-Specific Antigen Recurrence after Radical Prostatectomy or External-Beam Radiation Therapy for Clinically Localized Prostate Cancer. Journal of Clinical Oncology, 17, 168-172. https://doi.org/10.1200/JCO.1999.17.1.168

[11] Crook, J., Ots, A.F., et al. (2013) Prognostic Factors for Newly Diagnosed Prostate Cancer and Their Role in Treatment Selection. Seminars in Radiation Oncology, 23, 165-172. https://doi.org/10.1016/j.semradonc.2013.01.002

[12] Cooperberg, M.R., Broering, J.M., Carroll, P.R., et al. (2010) Time Trends and Local Variation in Primary Treatment of Localized Prostate Cancer. Journal of Clinical Oncology, 28, 1117-1123. https://doi.org/10.1200/JCO.2009.26.0133 
[13] Beckendorf, V., Guerif, S., Le Prise, E., et al. (2011) 70 Gy Versus 80 Gy in Localized Prostate Cancer: 5-Year Results of Getug 06 Randomized Trial. International Journal of Radiation Oncology Biology Physics, 80, 1056-1063. https://doi.org/10.1016/j.ijrobp.2010.03.049

[14] Dearnaley, D.P., Sydes, M.R., Graham, J.D., et al. (2007) Escalated-Dose Versus Standard-Dose Conformal Radiotherapy in Prostate Cancer: First Results from the MRC RT01 Randomised Controlled Trial. Lancet Oncology, 8, 475-487. https://doi.org/10.1016/S1470-2045(07)70143-2

[15] Ohri, N., Dicker, A.P., Showalter, T.N., et al. (2012) Late Toxicity Rates Following Definitive Radiotherapy for Prostate Cancer. Canadian Journal of Urology, 19, 6373-6380.

[16] Peeters, S.T.H., Heemsbergen, W.D., Koper, P.C.M., et al. (2006) Dose-Response in Radiotherapy for Localized Prostate Cancer: Results of the Dutch Multicenter Randomized Phase III Trial Comparing 68 Gy of Radiotherapy with 78 Gy. Journal of Clinical Oncology, 24, 1990-1996. https://doi.org/10.1200/JCO.2005.05.2530

[17] Pollack, A., Zagars, G.K., Starkschall, G., et al. (2002) Prostate Cancer Radiation Dose Response: Results of the M.D. Anderson Phase III Randomized Trial. International Journal of Radiation Oncology, Biology, Physics, 53, 1097-1105. https://doi.org/10.1016/S0360-3016(02)02829-8

[18] Cahlon, O., Zelefsky, M.J., Shippy, A., et al. (2008) Ultra-High Dose (86.4 Gy) IMRT for Localized Prostate Cancer: Toxicity and Biochemical Outcomes. International Journal of Radiation Oncology, Biology, Physics, 71, 330-337. https://doi.org/10.1016/j.ijrobp.2007.10.004

[19] Viani, G.A., Stefano, E.J., Afonso, S.L., et al. (2009) Higher-Than-Conventional Radiation Doses in Localized Prostate Cancer Treatment: A Meta-Analysis of Randomized, Controlled Trials. International Journal of Radiation Oncology, Biology, Physics, 74, 1405-1418. https://doi.org/10.1016/j.ijrobp.2008.10.091

[20] Dearnaley, D.P., Sydes, M.R., Langley, R.E., et al. (2007) The Early Toxicity of Escalated Versus Standard Dose Conformal Radiotherapy with Neo-Adjuvant Androgen Suppression for Patients with Localised Prostate Cancer: Results from the MRC RT01 Trial (ISRCTN47772397). Journal of Clinical Oncology, 83, 31-41. https://doi.org/10.1016/j.radonc.2007.02.014

[21] Daskivich, T.J., Chamie, K., Kwan, L., et al. (2011) Overtreatment of Men with Low-Risk Prostate Cancer and Significant Comorbidity. Cancer, 117, 2058-2066. https://doi.org/10.1002/cncr.25751

[22] Zietman, A.L., DeSilvio, M.L., Slater, J.D., et al. (2005) Comparison of Conventionaldose Vs High-Dose Conformal Radiation Therapy in Clinically Localized Adenocarcinoma of the Prostate: A Randomized Controlled Trial. Journal of the American Medical Association, 294, 1233-1239. https://doi.org/10.1001/jama.294.10.1233

[23] Pollack, A., Zagars, G.K., Smith, L.G., et al. (2000) Preliminary Results of A Randomized Radiotherapy Dose-Escalation Study Comparing 70 Gy with 78 Gy for Prostate Cancer. Journal of Clinical Oncology, 18, 3904-3911. https://doi.org/10.1200/JCO.2000.18.23.3904

[24] Saracino, B., Petrongari, M.G., Marziet, S., et al. (2014) Intensity-Modulated Pelvic Radiation Therapy and Simultaneous Integrated Boost to the Prostate Area in $\mathrm{Pa}$ tients with High-Risk Prostate Cancer: A Preliminary Report of Disease Control. Cancer Medicine, 3, 1313-1321. https://doi.org/10.1002/cam4.278

[25] Michalski, J.M., Yan, Y., Watkins-Bruner, D., et al. (2013) Preliminary Toxicity Analysis of 3-Dimensional Conformal Radiation Therapy Versus Intensity Modulated Radiation Therapy on the High-Dose Arm of the Radiation Therapy Oncol- 
ogy Group 0126 Prostate Cancer Trial. International Journal of Radiation Oncology, Biology, Physics, 87, 932-938. https://doi.org/10.1016/j.ijrobp.2013.07.041

[26] Arcangeli, S., Saracino, B., Petrongari, M.G., et al. (2007) Analysis of Toxicity in Patients with High Risk Prostate Cancer Treated with Intensity-Modulated Pelvic Radiation Therapy and Simultaneous Integrated Dose Escalation to Prostate Area. Radiotherapy and Oncology, 84, 148-155. https://doi.org/10.1016/j.radonc.2007.06.011

[27] Spratt, D.E., Zumsteg, Z.S., Zelefsky, M.J., et al. (2014) Comparison of High-Dose (86.4 Gy) IMRT Vs Combined Brachytherapy plus IMRT for Intermediate-Risk Prostate Cancer. BJU International, 114, 360-367. https://doi.org/10.1111/bju.12514

[28] Wortel, R.C., Incrocci, L., Pos, F.J., et al. (2015) Acute Toxicity After Image-Guided Intensity Modulated Radiation Therapy Compared to 3D Conformal Radiation Therapy in Prostate Cancer Patients. International Journal of Radiation Oncology, Biology, Physics, 91, 737-744. https://doi.org/10.1016/j.ijrobp.2014.12.017

[29] Volker, R.A., Nour, M. Hammoud, et al. (2016) Image-Guided Intensity-Modulated Radiotherapy of Prostate Cancer Analysis of Interfractional Errors and Acute Toxicity. Strahlentherapie und Onkologie, 192, 109-117. https://doi.org/10.1007/s00066-015-0919-y

[30] David, S., Pat, C., El-Gayed, A., et al. (2010) Planning Target Volume Margins for Prostate Radio Therapy Using Daily Electronic Portal Imaging and Implanted Fiducial Markers. Radiation Oncology Journal, 5, 52. https://doi.org/10.1186/1748-717X-5-52

\section{Submit or recommend next manuscript to SCIRP and we will provide best service for you:}

Accepting pre-submission inquiries through Email, Facebook, LinkedIn, Twitter, etc. A wide selection of journals (inclusive of 9 subjects, more than 200 journals)

Providing 24-hour high-quality service

User-friendly online submission system

Fair and swift peer-review system

Efficient typesetting and proofreading procedure

Display of the result of downloads and visits, as well as the number of cited articles

Maximum dissemination of your research work

Submit your manuscript at: http://papersubmission.scirp.org/

Or contact jet@scirp.org 